\title{
Erosion of the left ventricle by the epicardial patch of an automatic implantable cardioverter defibrillator
}

\author{
A G C Sutton, J M McComb, M A de Belder
}

Cardiothoracic

Division, South

Cleveland Hospital,

Marton Road,

Middlesbrough

TS4 3BW, UK

A G C Sutton

M A de Belder

Freeman Hospital, Freeman Road, High Heaton, Newcastle Upon Tyne NE7 7DN, UK

J M McComb

Correspondence to: Dr Sutton.

Accepted for publication 10 November 1998

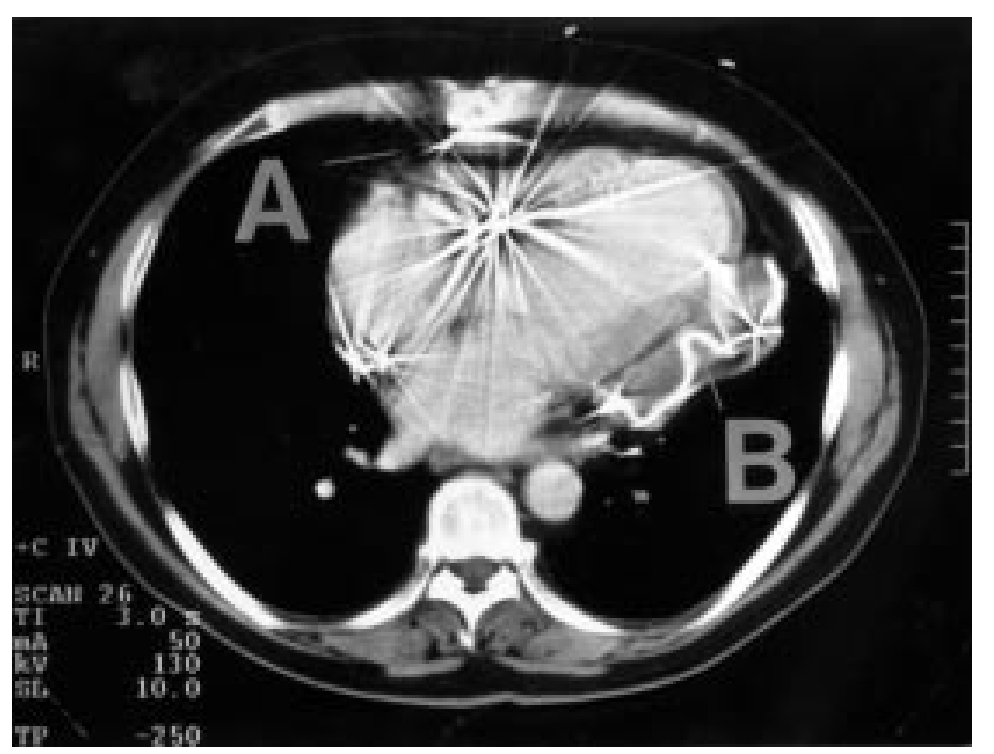

Figure 1 Computed tomography showing $(A)$ the anterior epicardial defibrillator patch with an apparent fracture, and $(B)$ the posterior epicardial defibrillator patch lying within the hypertrophied posterior left ventricular wall.

\begin{abstract}
A 56 year old man with an implantable cardioverter defibrillator was admitted with chest pain and collapse. Erosion of the left ventricle by an epicardial patch was confirmed by thoracotomy, but surgical repair was impossible. This rare complication should be considered in patients with a history of cardioverter defibrillators implanted by thoracotomy.

(Heart 1999;82:112-113)
\end{abstract}

Keywords: implantable cardioverter defibrillator; epicardial patch

In March 1988, the patient had a large anteroinferior myocardial infarction complicated by pulmonary oedema and ventricular tachycardia. In September 1990, he had coronary artery bypass grafting, with the implantation of a Teletronics Guardian defibrillator below the right superior rectus sheath. Two Teletronics defibrillator patches were anchored posteriorly on the left ventricle and anteriorly on the right ventricle, and a bipolar endocardial lead was positioned in the apex of the right ventricle through a right atrial pursestring suture. In 1994, the generator was replaced and a new transvenous sensing electrode was implanted. The original patches were used. In March 1996, a new transvenous pre-pectoral implantable cardioverter defibrillator (ICD) was inserted. The old abdominal generator and transvenous sensing lead were removed, but we decided that the risks of thoracotomy to remove the original epicardial patches outweighed the benefits of removing them. The leads were capped and buried in the abdominal pocket. In November 1996, the patient was admitted for excision of a granuloma and a prolene stitch from his abdominal wound.

In June 1997, three weeks before this admission, he was admitted for investigation of worsening dyspnoea. Computed tomography of the chest (fig 1) showed a $3 \mathrm{~cm}$ thick, soft tissue plaque rising from the posterior left ventricular wall. The posterior epicardial patch was in this plaque and was crumpled and fractured in places. There was no evidence of ventricular constriction to account for the dyspnoea, which was presumed secondary to the previously documented poor left ventricular function. Left and right cardiac catheterisation revealed no new or unexpected findings. In particular, there was no evidence of a leak on the left ventriculogram (fig 2). The patient was seen to have a small area of induration over the rectus sheath at the site of the previous granuloma excision, but there was no obvious infection. His symptoms improved after intravenous diuretic treatment and he was discharged.

On the most recent admission, the area of induration over the rectus sheath and the reappearance of a granuloma suggested chronic infection of the buried leads of the original defibrillator system. It seemed possible that ascending infection involving the epicardial patches had resulted in penetration of either the heart or a pulmonary vessel. Computed tomography of the thorax and upper abdomen confirmed that there was a large left pleural effusion with the characteristic appearance of blood, and a soft tissue plaque posterior to the heart containing the epicardial patch, as had been seen with computed tomography three weeks before. The abdominal cuts were consistent with infection around the old 


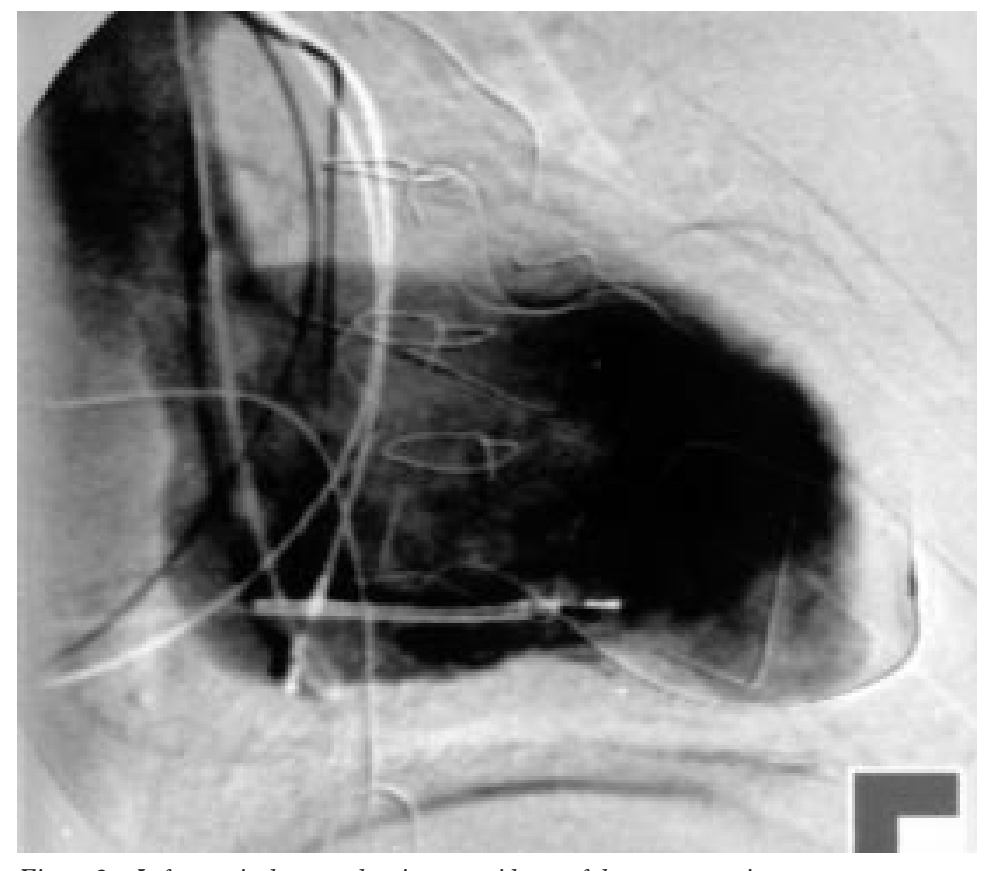

Figure 2 Left ventriculogram showing no evidence of dye extravasation.

abdominal wires. Cardiac catheterisation showed no evidence of leak from the left or right ventricle, no evidence of leak from any of the bypass anastomotic sites, and normal pulmonary arteries. Despite these findings, the clinical picture supported a diagnosis of erosion by an epicardial patch and we made the decision to attempt surgical repair.

Thoracotomy was performed through a left postero-lateral incision. During the operation, we found that three corners of the posterior epicardial patch had eroded through the pericardium and into the left lung (upper lobe, lingula, and medial lower lobe close to the hilum). The lower lobe was bleeding profusely. The lingula, upper lobe, and lower lobe were successfully dissected from the patch, and the tear in the lower lobe repaired. However, the fourth corner of the patch had eroded into the left ventricle and had been bleeding for some time, which was shown clearly by the marked thrombus formation. There was a $2 \mathrm{~cm}$ tear under the thrombus which could not be repaired, and the patient exsanguinated. No pus was found at operation. Blood cultures, swabs from the abdominal wall, and a portion of the pericardial patch removed at operation, all failed to grow any significant pathogens.

\section{Discussion}

Originally, ICDs were positioned using thoracotomy (as in this patient) with placement of two or three defibrillator patches either inside or outside the pericardium. Additional epicardial or transvenous sensing electrodes were used and all leads were tunnelled subcutaneously to the device, which was placed in an abdominal pocket. Implantation of ICDs by thoracotomy has a mortality of $3-5 \%$, and greater morbidity than transvenous implantation. ${ }^{1}$ Numerous complications following ICD implantation have been described, including penetration into the bronchial tree with resulting haemoptysis. ${ }^{2-4}$ Our patient, who also had haemoptysis, died from erosion of an epicardial defibrillator patch into the left ventricle. Although unproven, we believe that chronic low grade infection of the original defibrillator system may have contributed to his death.

We acknowledge the contribution of S Hunter, Consultant Cardiothoracic Surgeon, and Medical Illustration, of South Cleveland Hospital, Middlesbrough.

1 Zipes DP, Roberts D. Results of the international study of the implantable cardioverter defibrillator: A comparison of epicardial and endocardial lead systems. Circulation 1995;92:59-65.

2 Verheyden CN, Price L, Lynch DJ, et al. Implantable cardioverter defibrillator patch erosion presenting as haemoptysis. F Cardiovasc Electrophysiol 1994;5:961-3.

3 Lick SD, Conti VR. Automatic internal cardioverterdefibrillator patch erosion into the upper airway presenting as a cavitary lesion. Chest 1997;112:1144-6.

4 Dasgupta A, Mehta AC,Rice TW, et al. Erosion of implantable cardioverter defibrillator patch electrode into airways: able cardioverter defibrillator patch electrode into airways: 252-4. 